\title{
Integration of Multiple ECG Databases into a Unified Framework
}

\author{
MCJ de Wijs, M van Ettinger, SH Meij, SP Nelwan \\ Thoraxcenter - Erasmus MC-Rotterdam- The Netherlands
}

\begin{abstract}
Nowadays, due to the many different formats used by different manufacturers at the Thoraxcenter and the availability of only one ECG viewer in our Electronic Patient Information System, there is a need to convert the different formats to the format supported and to search for a (webbased) browser for our ECGs to eliminate the need to install and maintain all clients.

We developed a framework focussing on the recently approved SCP standard, to be independent of a manufacturer. This framework can convert SCP, Unipro, and Sifor to each other. Several viewers are available, but difficulties have arisen due to the many degrees of freedom in the earlier SCP versions. For the moment no new viewer has been introduced at our hospital.
\end{abstract}

\section{Introduction}

Since 1992, our hospital uses the ECG management system of Mortara (E-Scribe). More than 400.000 ECGs are now stored in this database. In Patien98 [1], our electronic patient health record (2000 clients installed hospital wide), all these ECGs can be viewed with a locally installed Mortara fat-client viewer.

Nowadays, multiple applications generate 12 lead ECGs such as our bedside monitors (Dräger, Megacare) and stress test systems (Welch\&Allyn/ CardioControl (WACC)); all with their own proprietary formats and viewers: MDW (WACC), Unipro (Mortara), and Sifor (Dräger). Only WACC supports other formats beside their own: SCP and Sifor (Table 1).

\begin{tabular}{|l|l|l|}
\hline & Manufacturer & Formats \\
\hline Diagnostic ECG & Mortara & Unipro \\
\hline Bedside monitoring & Dräger & Sifor \\
\hline $\begin{array}{l}\text { Diagnostic ECG + } \\
\text { Stress }\end{array}$ & Welch\&Allyn & $\begin{array}{l}\text { Sifor, SCP, } \\
\text { MDW }\end{array}$ \\
\hline
\end{tabular}

\section{Table 1. Overview of different formats}

Our objective is to use only one viewer for all 12-lead ECGs in Patien98. To get rid of the maintenance and installing of fat-clients throughout the hospital, the viewer should be web based.

\section{Methods}

In order to achieve our objective to be able to use one viewer, we developed a framework that converts the different ECG-formats visa versa. We focused on the SCP-ECG standard, recently approved by CEN/(EN 1064) and ANSI/AAMI (EC71)[2]. SCP was intended to define a uniform format for processing, communicating, and storing of electronic ECG records. To our advantage the SCP and Unipro format have many similarities [3].

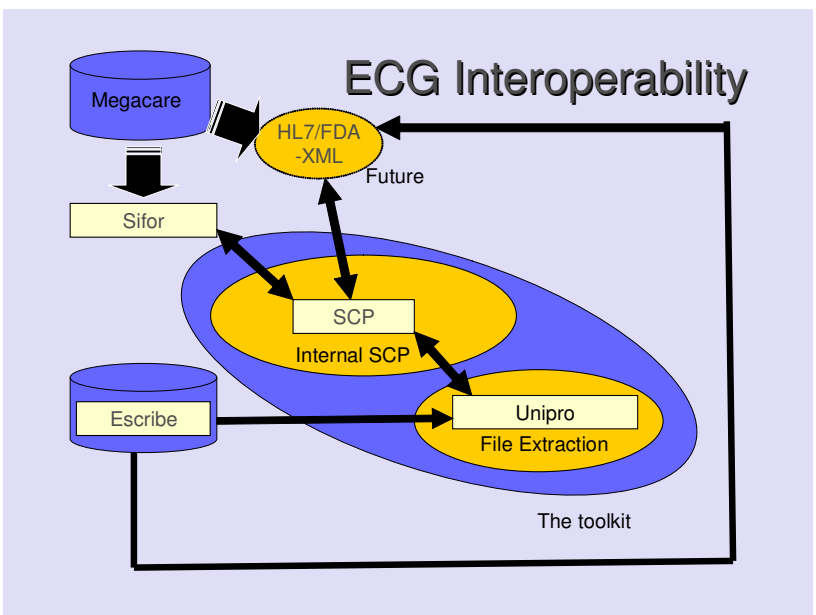

Figure 1. ECG Interoperability, an overview of the different translation routes.

To be able to view all three formats with one single viewer, we developed a tool in $\mathrm{C \#}$ and the Microsoft DOT NET framework on a windows platform by using the libraries of the manufacturers. Internally the framework utilizes the specification of SCP data interchange format. All export and import operations convert to and from this data format, in all cases no manufacturer library is available (see Figure 1).

\section{Results}

We have three executables that convert one format to another, covering all formats (see Figure 2). All are command line, enabling batch jobs and which can be run from another application, e.g. a web application. One tool 
extracts the Sifor file from the Megacare database and stores it onto disk. Another tool uses the WACC libraries to convert from Sifor to SCP or vice versa. The last one is able to do both the extracting of the Unipro files from the E-Scribe database and the conversion of SCP to Unipro or back. However, as with Sifor/Megacare, it isn't able to put exported Unipro files into the E-Scribe database.

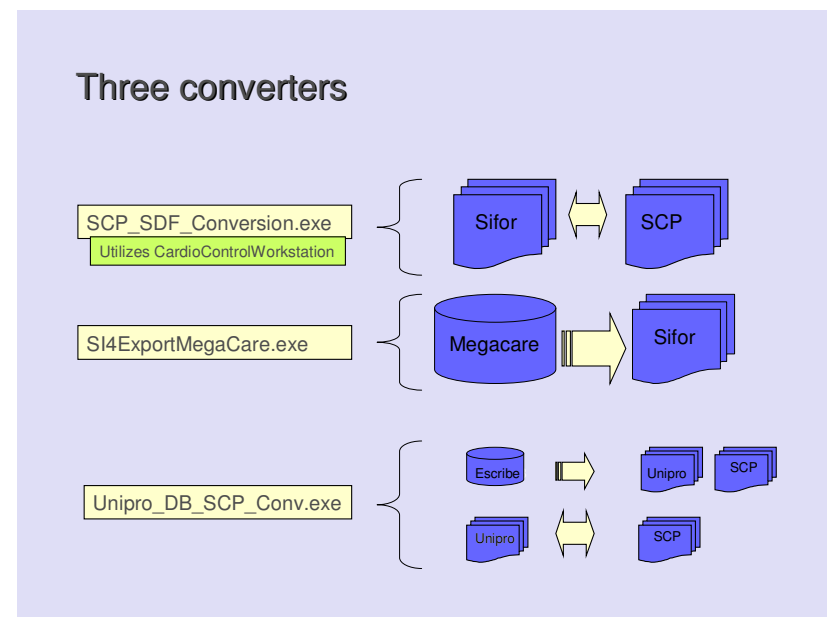

Figure 2. Three converters, a list of the tools and the translation they process.

Both WACC and ICS-FORTH have tested our SCP files. We made use of both the web based and the email testing service of the OpenECG community [4]. The generated SCP files were SCP 2.0 compliant, confirmed by ICS-FORTH and WACC (Figure 5, Figure 7). The tests done by the two OpenECG services were not completely valid. They haven't been upgraded to SCP 2.0 and still operate on 1.3 and 1.4, resulting in errors during parsing of sections 1,7 , and 10 . The leads could be decoded as shown in Figure 7.

We were able to view our ECGs in all our ECG viewers, except Megacare because there is no import (see Figure 3). WACC does support the Sifor format and be viewed, but it isn't possible to upload into Megacare. Our generated Unipro files can be viewed by Mortara EScribe viewer (Figure 5).

We succeeded into converting 400.000 E-Scribe ECGs to SCP, with a failure rate of about $0.1 \%$. Most failures were caused by wrong settings (median substracting turned on). Without this, there were almost no errors.

However, we did encounter several other problems:

- Some items were not present in one of the formats. If this was the case, defaults were applied.

- The sample rate between formats was different (one time based, the other frequency based). Polynomial re-sampling was done, utilizing algorithms from Numerical Recipes for C [5], converted to C\#.
- $\quad$ Both WACC and the ICS-FORTH (Figure 4, Figure 6) made the assumption of only 8 leads (I, II, V1-6) present when decoding the SCP in their viewers, whereas Sifor uses 12 leads, causing decoding problems in both viewers with SCP files generated from Sifor.

- Some of the Unipro files have a length of only $7 \mathrm{~s}$, instead of $10 \mathrm{~s}$.
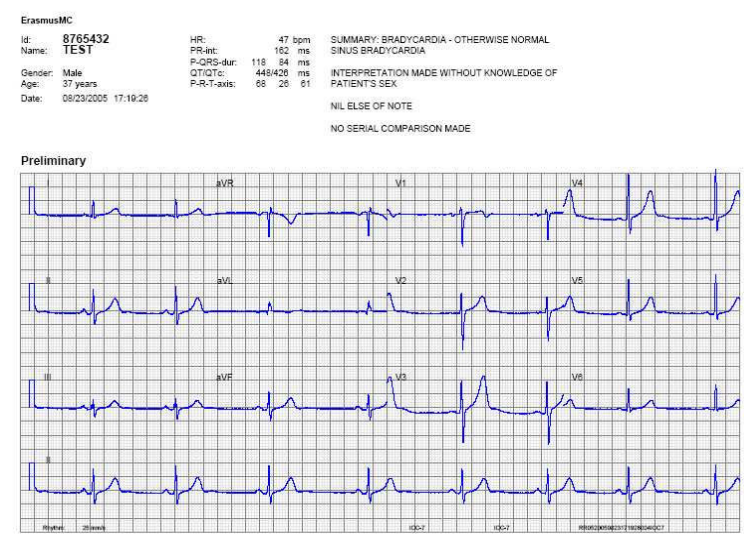

Figure 3. Dräger, ECG recorded with Infinity, viewed with Megacare.

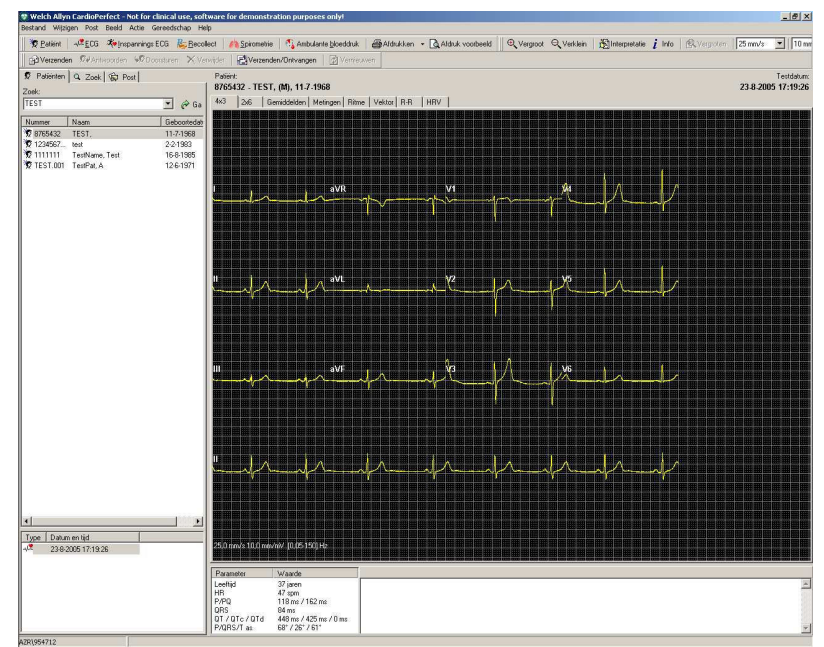

Figure 4. WACC, the ECG recorded with Infinity, converted to SCP, viewed by WACC. 


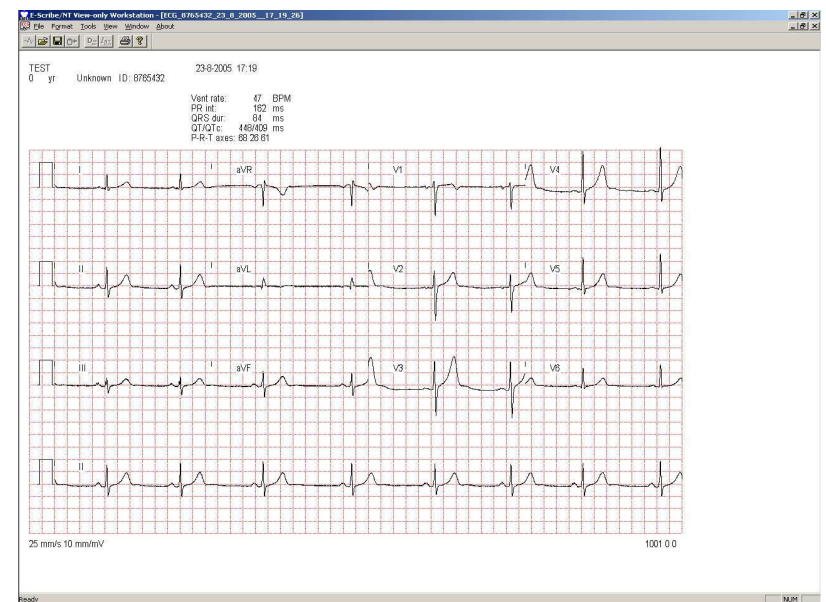

Figure 5. E-Scribe, the ECG recorded with Infinity, via SCP, to UNIPRO and viewed by E-Scribe

\section{Discussion}

We partially succeeded in our objective of having one viewer; the current E-Scribe viewer can be used for all ECGs. In the future this will be replaced by the web based Mortara Athena Viewer. Together with our framework, the objective will be fulfilled.

With this new framework, one is free to use any viewer supporting one of the described formats for the viewing of ECGs. By using SCP internally it is easy to export/import from other formats, as long as the conversion to and from SCP is documented or available as a library.

We tested a few currently available SCP-ECG viewers and they all have version or interpretation problems. Most of the problems are related to the different interpretations of the early 1.3 and 1.4 SCP standard. In SCP 2.0 a big effort has been made to omit these different interpretations, but as mentioned previously, some viewers still assumes a fixed number of 8 leads whereas the SCP standard is clearly capable to store an arbitrary number of leads. It can be argued that 8 leads are enough to reconstruct all 12, but that limit is not defined in SCP.

One solution could be an OpenSource SCP-ECG viewer that supports the different SCP versions or interpretations and is invulnerable to the different interpretations of the earlier standard. Another solution may be the HL7/FDA-XML standard based upon the HL7 Version 3 [6]. Its application is growing; hence a web based version is likely to be developed in the near future [7].

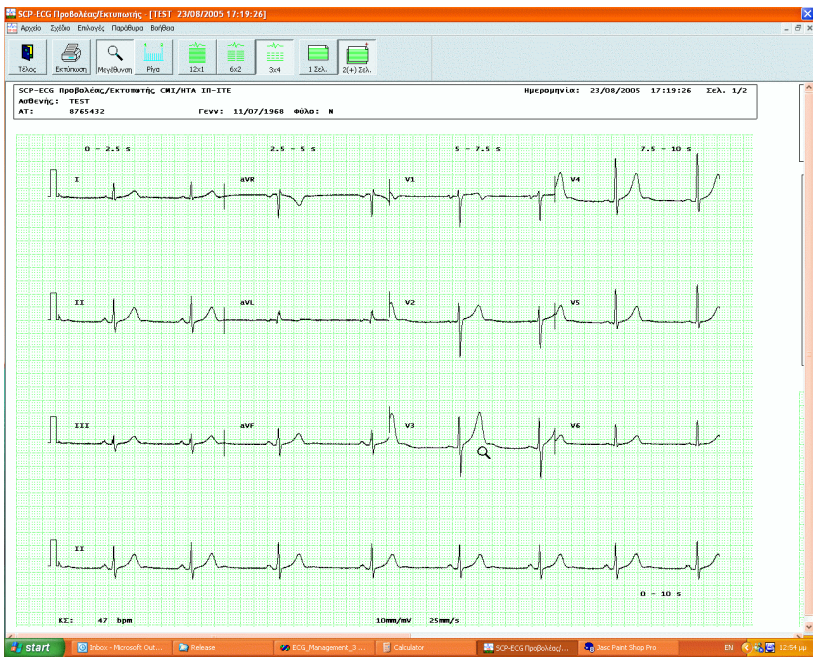

Figure 6. Forth-SCP viewer, the ECG recorded with Infinity, converted to SCP, viewed by Forth.

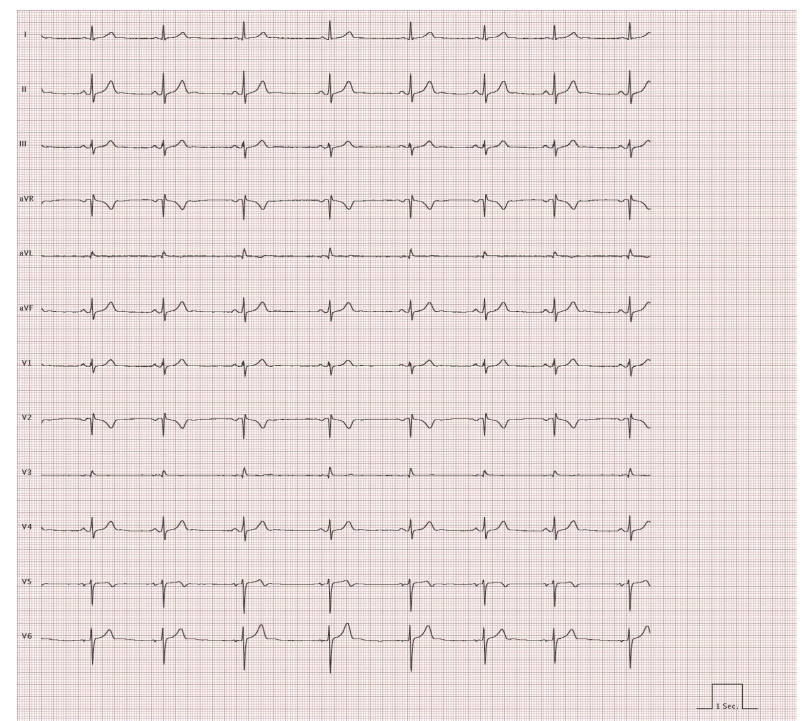

Figure 7. OpenECG SCP parsing server result

\section{Acknowledgements}

The authors would like to thank Mr. Lakamp (WACC) and Mr. Chiarugi (CMI-HTA, FORTH) for their help and cooperation.

\section{References}

[1] N van der Putten, SP Nelwan etc. Patient 98: a Componentbased Multimedia Workstation for Cardiac Care and 
Research. Computer in Cardiology 1999:26; 77-80.

[2] ENV 1064 standard communication protocol for computerassisted electrocardiography, European Committee from Standardization, Brussels, Belgium 1996.

[3] F Chiarugi, PJ Lees, CE Chronaki etc. Developing Manufacturer-Independent Components for ECG Viewing and for Data Exchange with ECG Devices:Can the SCPECG Standard Help? Computers in Cardiology 2001; 28:185-188.

[4] www.openecg.net.

[5] Numerical recipes in C, books online, chapter 3 http://www.library.cornell.edu/nr/bookcpdf.html

[6] Health Level Seven Version 3 Standard Ballot Package, [30 juli 2004]. Available at http://www.hl7.org/v3annecg/.

[7] Digtial ECG XML Viewer available at: http://www.ampsllc.com/.

Address for correspondence

MCJ de Wijs, MSc

Office BA569

Thoraxcenter, ErasmusMc

Postbus 2040,

3000 CA Rotterdam

M.deWijs@ErasmusMc.nl 